\title{
RobotEye Technology for Thermal Target Tracking Using Predictive Control
}

\author{
Ansu Man Singh ${ }^{1}$, Q. P. Ha ${ }^{1}$, D. K. Wood ${ }^{2}$, M. Bishop ${ }^{2}$, Q. Nguyen ${ }^{3}$ and A. Wong ${ }^{3}$ \\ 1.School of Electrical, Mechanical and Mechatronic Systems, University of Technology Sydney, Australia \\ 2. Ocular Robotics Pty Ltd, NSW, Australia \\ 3. Defence Science and Technology Group (DSTG), Department of Defence, Australia
}

\begin{abstract}
-
This paper presents a system for tracking thermal targets using the RobotEye technology. The system comprises of a thermal camera, a vision camera, a RobotEye, and a fiducial detection system. A marker is attached to a thermal target in order to estimate its position and orientation using the marker detection system. Then, an estimator predicts the future position of the target. A predictive control based on the Model Predictive Control (MPC) approach is then applied to generate commands for the eye to follow the target. Results of the tracking by MPC are also presented in this paper along with the performance evaluation of the whole system. The evaluation clearly shows the improvement in the tracking performance by the proposed system. Keywords -
\end{abstract}

Automation and control, Computer Vision

\section{Introduction}

In the last few decades, due to the advancement of the state-of-the-art technology, thermal vision systems find application in many areas [1]. For example, in [2] such systems are used to detect faults in the electrical transmission lines which can save millions of dollars in maintenance of power systems. Nevertheless, such sensors are quite handy in the stress analysis of structures, which is also known as thermoelastic stress analysis.

Thermoelastic stress analysis (TSA) is a non-contact method for stress measurements. The method is based on thermoelastic effect which shows a relationship between the change in temperature of solid structure and its deformation [3]. This technique is widely used in aerospace industries for the maintenance and structural health monitoring of aircraft [4].

Many studies have reported that sensors used in TSA application consist of uncooled and cooled type. Formal imagers, also known as microbolometers, are compact, less expensive, and do not require an external agent to maintain their temperature. On the other hand, cooled type requires a coolant such as nitrogen to regulate the temperature of the instrument. A comparative study of both sensor types is provided in [5], and it clearly reveals that uncooled sensors are outperformed by microbolometers in stress analysis, even though they have high sensitivity.

For the TSA, the infrared sensors are placed in a fixed location with respect to the subject of interest. However, one of the disadvantages of such approaches arises due to the motion of the dynamic objects. As a result of the motion of object-under-test, the thermal images become blurred and hence analysis is obscured.

One solution of the above-mentioned issue is the use of a pan-tilt system to hold the infrared cameras. For instance, in [6] such systems are used in the thermoelastic analysis of airframe to track the test specimen and compensate its motion. Similarly, they can also be used with unmanned aerial vehicles (UAV) to track the motion of objects such as ships, as in [8]. However, since the cameras are attached to the pan-tilt system, the mass and inertia of the system increases and thus reducing the performance of the system, particularly when the speed of the test subject is much faster.

To enhance the performance, this paper presents a solution which was proposed by Defence Science and Technology Group for the application of TSA of aircraft. The prototype utilizes RobotEye, a state-of-the-art pointing technology which has been developed and commercialized by Ocular Robotics Pty Ltd [9]. The solution also comprises of a high-speed vision camera, a thermal camera, and a marker detection system. The thermal camera is embedded inside the RobotEye and to capture the view where the eye is pointing. RobotEye uses mirror-based technology [10] which significantly reduces the inertia of the sensor in comparison to the pan-tilt system. As a result, the tracking performance can be considerably improved.

In the system, a fiducial marker is attached to the target, and its position is calculated using the marker detection system, as presented in [11], from the images captured by the vision camera. The marker detection system has the latency of less than $10 \mathrm{~ms}$ which is ideal for high-speed tracking applications. 


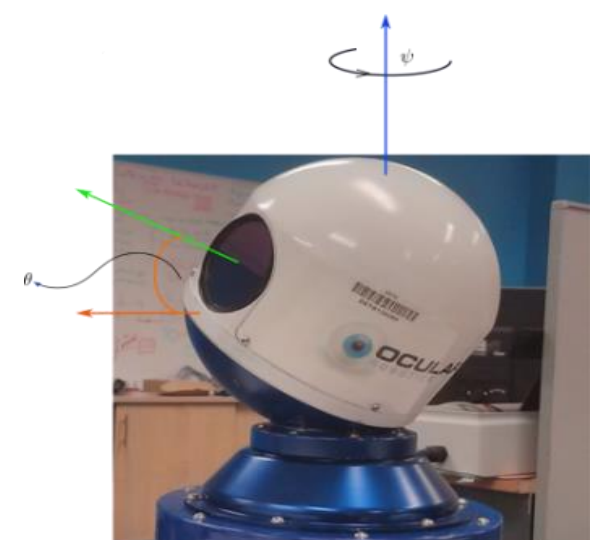

Figure 1: Variables representing robot eye head motion

Moreover, this paper contributes to the design of a predictive tracking controller based on the Model Predictive Control (MPC) for RobotEye head motion. Since the eye is connected to a computer network, its tracking performance is affected by delays. Similarly, delays are also common while processing images from vision cameras. Nevertheless, owing to the MPC, the control system for the actuator can generate control signals even in the presence of the unexpected delays.

The significance of this study is not only in the modeling and control system but also in the TSA based fatigue analysis of structures. As mentioned previously, mechanical structures that are under constant stress and strain show increments in the surface temperature, which are the good indicators of failure points. Since the proposed system can be used in tracking thermal targets even at high speed, it can have a huge advantage in such applications.

This paper is arranged in following sections. Details about the RobotEye technology and the proposed system are presented in Section 2, followed by the MPC for the robot eye head motion in Section 3. Results are presented in Section 4. Finally, this paper concludes with a conclusion and recommendation for future works.

\section{RobotEye Technology}

The RobotEye has an advantage in its own cuttingedge precision and responsiveness: it can enhance the mapping quality when used with laser sensors. In addition, the technology can be integrated with various kind of sensors such as thermal and hyper-spectral vision that can add an extra dimension to sensing technologies [9].

\subsection{Variables representing RobotEye gazing direction}

Orientation of the pointing device or its the gazing direction can be described by two variables namely azimuth $(\psi)$ and elevation $(\theta)$ as shown in the Figure 1. The azimuth angle represents the rotation of the head about the vertical axis as depicted in the figure. Similarly, the elevation angle represents the angle made by the viewing direction with respect to the horizontal plane.

\subsection{Thermal target tracking using RobotEye}

The advantage of light weight design makes the RobotEye technology a prefered choice in tracking applications. The laboratorial set-up for this application

consists of a high-speed vision camera, a robot eye, a moving thermal target, a circular patches marker, and a thermal camera.

In the thermal target tracking system, the target is placed at the center of a fiducial marker which is within the field-of-view of the vision camera. The marker consists of circular patches. Details on the marker design and the detection algorithm are provided in [11]. Similarly, the thermal camera is embedded inside the robot eye, thus, it can capture the view where the robot eye is pointing at. This system can have a huge implication in the fatigue analysis of mechanical which are under constant motion. For example, in [1], it is reported that fatigue analyses of such moving structures are adversly affected due to the blurry images captured by static cameras.

\subsection{System Configuration}

Coordinate systems assigned to the RobotEye and the vision camera for tracking are shown in Figure 2. The reference frames for the vision and the sensor are represented by $O_{V}$ and $O_{R}$, respectively. Similarly, the $R_{V}^{R}$ is the rotational matrix that represents the orientation of the robot with respect to the vision camera reference frame. Consider an observation of the target position with respect to the vision camera be represented as $p_{V}^{t}$. 
Then, the observation can be represented with respect to the robot reference frame as

$$
p_{R}^{t}=R_{V}^{R} p_{R}^{t}+p_{R}^{V}
$$

where $p_{R}^{t}$ is the position of target and $p_{V}^{t}$ is the location of vision camera with respect to the pointing device.

As presented in Section 2.1, gazing direction for the robot eye is represented by azimuth $\psi$ and elevation $\theta$ angles which are in the spherical coordinate system. Therefore, for the representation of the thermal target as the gazing direction from RobotEye, conversion from Cartesian to spherical coordinates should be applied as

$$
\begin{aligned}
& \psi=\tan ^{-1} \frac{y}{x} \\
& \theta=\tan ^{-1}\left(\frac{\sqrt{y^{2}+x^{2}}}{z}\right),
\end{aligned}
$$

where $p_{R}^{t}=\left[\begin{array}{lll}x & y & z\end{array}\right]$.

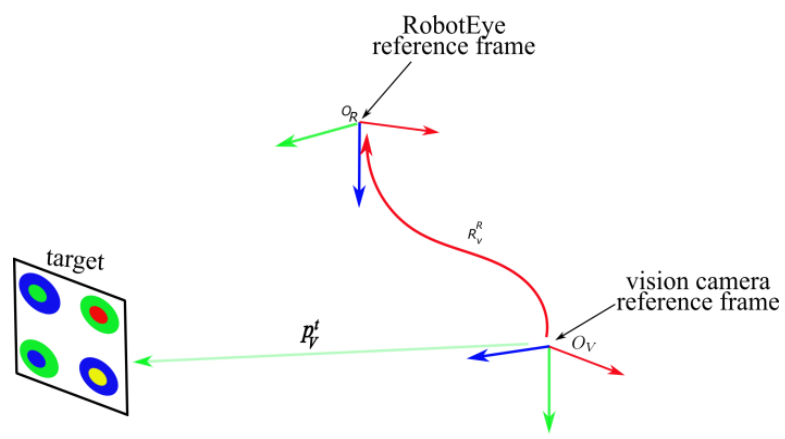

Figure 2. Arrangement of RobotEye and vision camera

\subsection{System architecture for thermal tracking system}

The system architecture for the proposed system comprising of a RobotEye, vision camera, and thermal camera is shown in Figure 3. In the system, the position and orientation of the target is estimated from the frames captured by vision camera which utilizes the circular marker detection algorithm [13].

After estimating the position of the thermal target with respect to the vision camera, it is calculated with respect to the eye in the azimuth and elevation space using equations (1) and (2). The conversion requires the extrinsic parameters such as $R_{V}^{T}$ and $p_{R}^{V}$. After that, a prediction algorithm based on Kalman filter is utilised to predict its future trajectory. The filter uses a constant velocity model to represent the target motion. The model is represented as

$$
\begin{array}{cl}
\boldsymbol{x}[n+1] & =A \boldsymbol{x}[n], \\
\boldsymbol{y}[n] & =\boldsymbol{C} \boldsymbol{x}[n],
\end{array}
$$

where $\boldsymbol{x} \in \mathbb{R}^{4}=[\psi \theta \dot{\psi} \dot{\theta}], \boldsymbol{y} \in \mathbb{R}^{2}=[\psi \theta]$,

$$
\begin{aligned}
A & =\left[\begin{array}{llll}
1 & 0 & T & 0 \\
0 & 1 & 0 & T \\
0 & 0 & 1 & 0 \\
0 & 0 & 0 & 1
\end{array}\right], \\
B & =\left[\begin{array}{llll}
1 & 0 & 0 & 0 \\
0 & 1 & 0 & 0
\end{array}\right],
\end{aligned}
$$

and $T$ is the sample period.

Finally, the predicted trajectory is fed to a model predictive controller (MPC) which generates commands for the robot driving systems. Here, the objective of MPC is to compensate for the lags in the system which are introduced through various sources such as sensing and network delays, in order to improve the overall performance of the system.

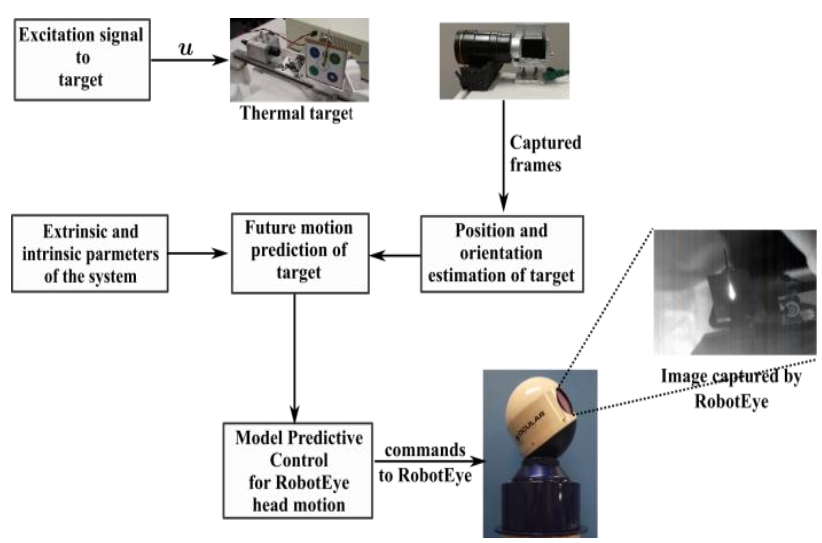

Figure 3. System architecture.

\section{Modelling and Control System Design}

\subsection{Modelling}

For the representation of the dynamics of the motion of sensor head motion, we have considered the statespace model in discrete-time as

$$
\begin{array}{ccc}
\boldsymbol{x}[n+1] & = & A \boldsymbol{x}[n]+B \boldsymbol{u}[n], \\
\boldsymbol{y}[n] & = & C \boldsymbol{x}[n],
\end{array}
$$

where, $\boldsymbol{x} \in \mathbb{R}^{n}, \boldsymbol{u} \in \mathbb{R}^{m}, \boldsymbol{y} \in \mathbb{R}^{p}$ are, respectively, the states, inputs and outputs of the system. In the case of the pointing actuator $\boldsymbol{y}=[\psi \theta]^{\mathrm{T}} \in \mathbb{R}^{2}$ and $\boldsymbol{u}=$ $\left[\psi_{c m d} \theta_{c m d}\right]^{\mathrm{T}}$, where $\psi_{c m d}$ and $\theta_{c m d}$ represent the command inputs to the system.

\subsection{System Identification}

For the identification of the system's parameter of Equation 4, we measured the angles and inputs through the application programming interface (API) provided 
by the robot. Then, we applied a standard identification algorithm such as N4SID in the framework provided by Matlab. Figure 4 shows the validation result of the identified method. The system matrices of the identified model are

$$
A=\left[\begin{array}{cccc}
-0.4141 & 288.6 & -848.2 & 54.97 \\
-75.28 & -40.89 & -495.1 & -127 \\
757.5 & 43.73 & -546.4 & -80.56 \\
75.16 & 48.69 & -210.6 & -13.13
\end{array}\right]
$$

$$
B=\left[\begin{array}{cc}
-96.69 & -82.96 \\
-42.41 & -45.76 \\
-108.7 & -92.47 \\
-28.4 & -24.58
\end{array}\right]
$$

and

$$
C=\left[\begin{array}{cccc}
-9.633 & -9.392 & -8.936 & 72.69 \\
19.64 & -11.09 & 7.066 & -98.29
\end{array}\right] \text {. }
$$

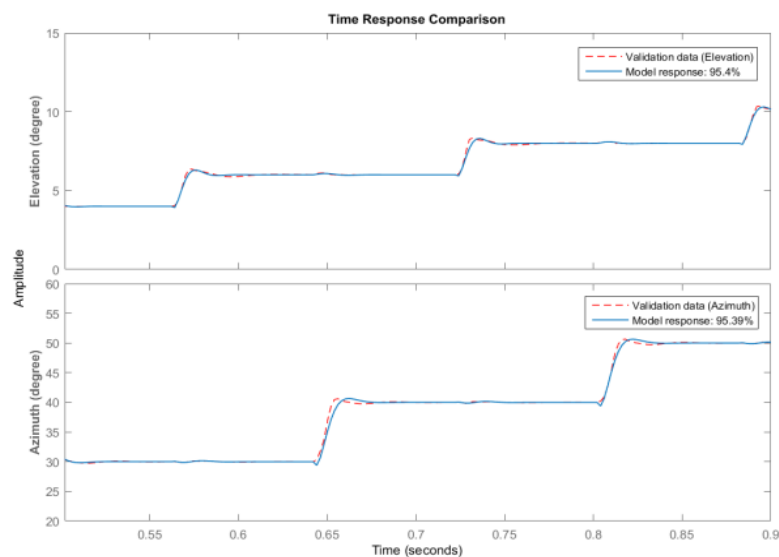

Figure 4. Validation of the identified model.

\section{Model Predictive Control (MPC)}

In order to apply the predictive control, we utilized the MPC in tracking the output reference signal. The problem is derived as

$\min \left\{\begin{array}{c}\frac{1}{2} \sum_{i=1}^{N}\left\{\left(\boldsymbol{y}_{i}-\boldsymbol{r}_{i}\right)^{\mathrm{T}} \boldsymbol{Q}_{\boldsymbol{y}}\left(\boldsymbol{y}_{i}-\boldsymbol{r}_{i}\right)+\boldsymbol{u}_{i}^{\mathrm{T}} \boldsymbol{Q}_{u} \boldsymbol{u}_{i}\right\} \\ +\left(\boldsymbol{y}_{N}-\boldsymbol{r}_{N}\right)^{\mathrm{T}} \boldsymbol{Q}_{N}\left(\boldsymbol{y}_{N}-\boldsymbol{r}_{N}\right)\end{array}\right\}$

subject to: $\left\{\begin{array}{c}\boldsymbol{u}_{\min } \leq \boldsymbol{u} \leq \boldsymbol{u}_{\max } \\ \boldsymbol{x}_{i+1}=A \boldsymbol{x}_{i}+B \boldsymbol{u}_{i}, \\ \boldsymbol{y}_{i}=C \boldsymbol{x}_{i},\end{array}\right.$

where $\boldsymbol{y}_{i}=\boldsymbol{y}[n+i]$ is the $i^{\text {th }}$ future output of the system, $\boldsymbol{u}_{i}=\boldsymbol{u}[n+i]$ is the $i^{\text {th }}$ future input to the system, $\boldsymbol{r}_{i}$ is the $i^{\text {th }}$ future reference, $N$ is the prediction horizon, $A$ is the system matrix, and $B$ is the input matrix. The cost function $V(\boldsymbol{x})$ can also be represented as

$$
\begin{gathered}
V(\boldsymbol{x})=\quad\left(\boldsymbol{y}_{0}-\boldsymbol{r}_{0}\right)^{\mathrm{T}} \boldsymbol{Q}_{0}\left(\boldsymbol{y}_{0}-\boldsymbol{r}_{0}\right) \\
+(\boldsymbol{Y}-\boldsymbol{R})^{\mathrm{T}} \overline{\boldsymbol{Q}}_{y}(\boldsymbol{Y}-\boldsymbol{R}) \\
\boldsymbol{U}^{\mathrm{T}} \overline{\boldsymbol{Q}}_{u} \boldsymbol{U}
\end{gathered}
$$

where

and

$$
\boldsymbol{Y}=\left[\begin{array}{c}
\boldsymbol{y}_{1} \\
\boldsymbol{y}_{2} \\
\vdots \\
\boldsymbol{y}_{N}
\end{array}\right], \boldsymbol{X}=\left[\begin{array}{c}
\boldsymbol{u}_{0} \\
\boldsymbol{u}_{1} \\
\vdots \\
\boldsymbol{u}_{N-1}
\end{array}\right]
$$

$$
\boldsymbol{R}=\left[\begin{array}{c}
\boldsymbol{r}_{1} \\
\boldsymbol{r}_{2} \\
\vdots \\
\boldsymbol{r}_{N}
\end{array}\right]
$$

Now, by utilising the system dynamics and forward substitution one can get

$$
\begin{aligned}
& \text { minimize } V(\boldsymbol{U})=\boldsymbol{U}^{\mathrm{T}} \bar{H} \boldsymbol{U}+\bar{F}^{\mathrm{T}} \boldsymbol{U} \\
& \text { subject to: } E \boldsymbol{U} \leq \boldsymbol{\delta}
\end{aligned}
$$

where

$$
\begin{aligned}
& \bar{H}=\bar{S}^{\mathrm{T}} \bar{Q} \bar{S}+\boldsymbol{R}, \\
& \bar{F}^{\mathrm{T}}=2 \boldsymbol{x}_{0} \bar{T} \bar{Q} \bar{S}-\boldsymbol{R}^{\mathrm{T}} \bar{Q} \bar{S},
\end{aligned}
$$

$$
\bar{E}=\left[\begin{array}{c}
I \\
-I \\
\bar{S} \\
-\bar{S}
\end{array}\right]
$$

and,

$$
\boldsymbol{\delta}=\left[\begin{array}{c}
\Delta \boldsymbol{U}_{\max } \\
-\Delta \boldsymbol{U}_{\min } \\
\boldsymbol{Y}_{\max }-\bar{T} \boldsymbol{x}_{\mathbf{0}} \\
-\boldsymbol{Y}_{\min }+\bar{T} \boldsymbol{x}_{\mathbf{0}}
\end{array}\right]
$$

Now, this problem can be solved using the available QP solver.

\section{Tracking results}

\subsection{Simulation}

Figure 5 shows the results of the MPC controller for the sinusoidal reference signal. From the figure it is clear that the controller is able to track the reference signal. Similarly, from the plots of error signals, as presented in Figure 6, it is clear that the error in the Azimuth space is less than 0.03 degrees, compared to 0.01 degrees in case of the elevation angle.

The tracking performance can also be evaluated in terms of the performance indices Integral square error (ISE) which is defined as:

$$
I S E=\sum_{i=1}^{M} e^{2}[n] .
$$


The ISE errors for Azimuth and Elevation angles are 0.34937 and 0.017448 degree $^{2}$, respectively.
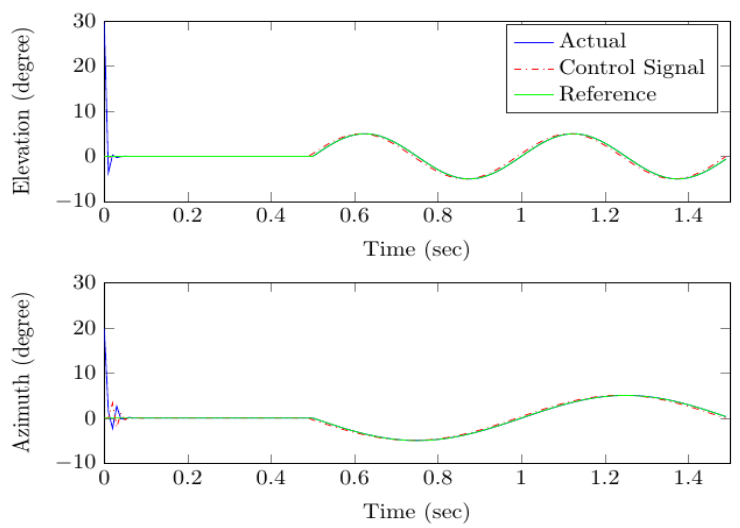

Figure 5. Plot of the angles along with command and actual values
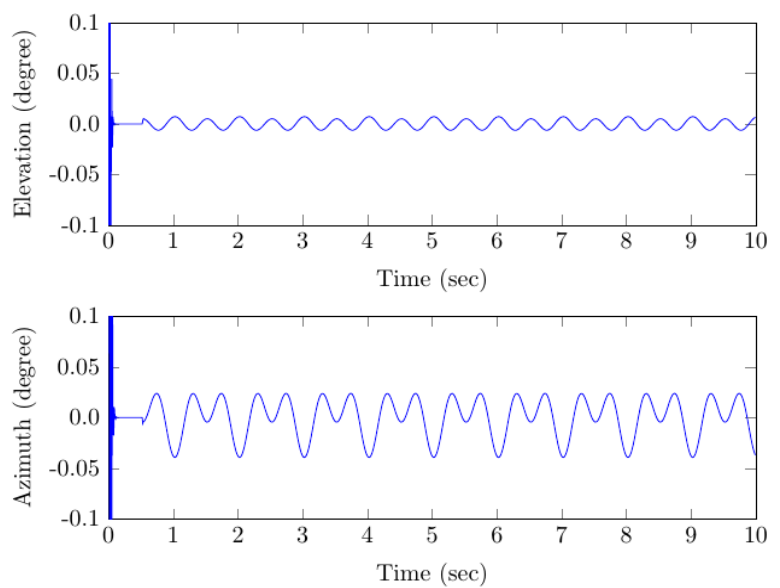

Figure 6. Plot of errors in Azimuth and Elevation angles

\subsection{Real time tracking results}

For the real-time testing, the MPC based predictive control algorithm was applied to a RobotEye with the specifications provided in Table 1 . The block diagram for the experiment is provided in the Figure 7.

In the experiment, sinusoidal signals were provided as the reference signals. The frequencies of the reference signals were $1 \mathrm{~Hz}$ and $2 \mathrm{~Hz}$ for azimuth and elevation angles, respectively. To send the angular commands and receive the measurements we used the proprietary application programming interface.

The tracking errors during the experiment are shown in Figure 8. The error is less than 0.2 degrees for elevation and 0.1 degrees for the azimuth angle. Similarly, ISE errors for the azimuth and elevation angles are 3.947 and 33.73 degree $^{2}$, respectively.

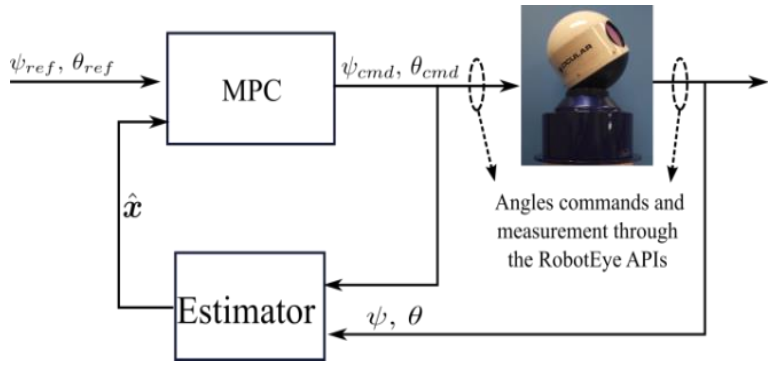

Figure 7: Real time experiment for MPC.
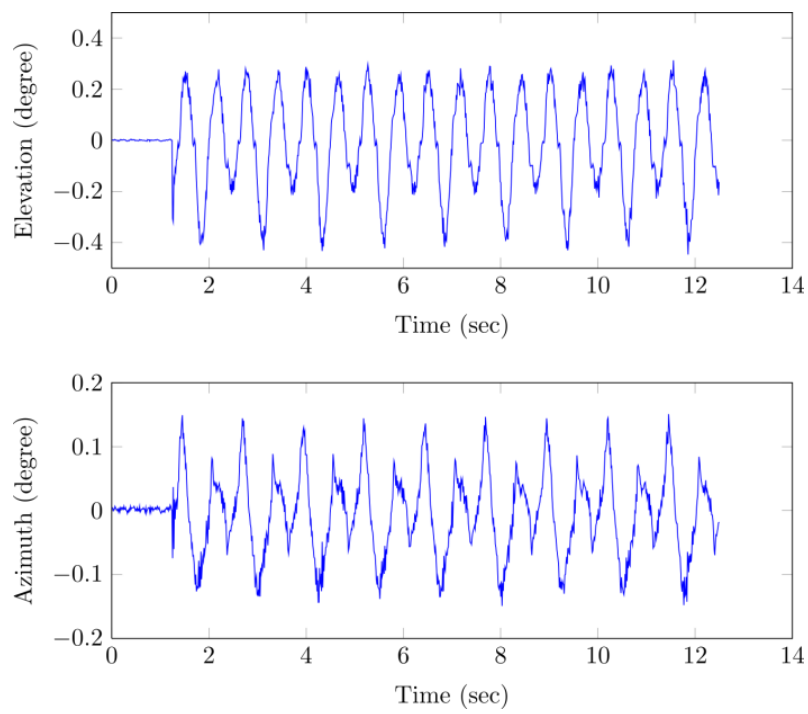

Figure 8. Tracking errors in azimuth and elevation angles in real experiment.

\subsection{Performance evaluation for thermal target tracking}

In this section, we evaluate performance of the proposed system described above. In addition, this section also presents comparison of the performance with and without the predictive control. The experimental setup for the evaluation of the system is presented in the Figure 9 with the specifications of all the components in Table 1.

The figure also shows the target consisting of the circular patched marker with a heat source at the centre of the marker. The heat source was used to detect the target in the thermal camera.

In this real time application, there is no direct way of evaluating the tracking performance of the system. However, as mentioned previously, the thermal camera is embedded inside RobotEye, and it has the view wherever it is pointing at. Therefore, the thermal targets are detected in the images of taken by thermal and vision cameras, and then compared for evaluation. 


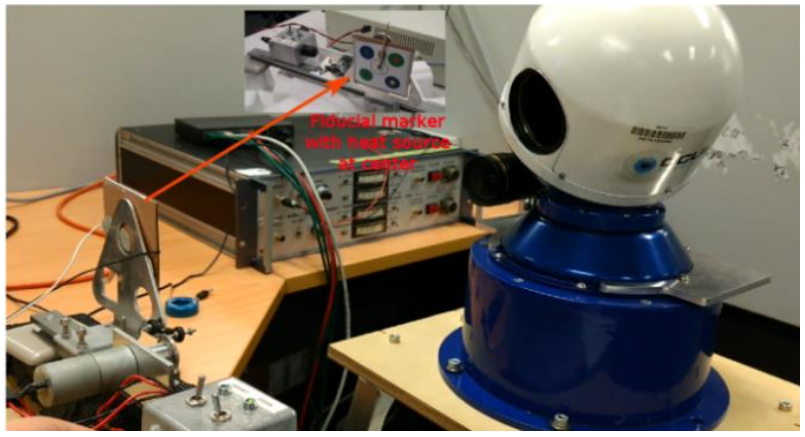

Figure 9: Experimental setup for tracking system.

The calculation of target pixels in the vision camera is straight forward because the circular fiducial marker is attached with the target. However, the calculation of the target pixels in the thermal images is not straight forward. Therefore, a thresholding process is applied in every image, followed by a blob detection algorithm. The purpose of the blob detection algorithm is to detect hot spot on the image which is the location of the target.

Table 1: Parameters of the system.

\begin{tabular}{ccc}
\hline & Parameter & Values \\
\hline \multirow{3}{*}{ Vision camera } & Focal length & $16 \mathrm{~mm}$ \\
& Resolution & $658 \times 492$ \\
& Frame Rate & $100 \mathrm{fps}$ \\
\hline \multirow{3}{*}{ Thermal camera } & Focal length & $19 \mathrm{~mm}$ \\
& Resolution & $640 \times 512$ \\
& Frame Rate & $30 \mathrm{fps}$ \\
\hline \multirow{2}{*}{ RobotEye } & Model no & RELW 50 \\
& Aperture diameter & $50 \mathrm{~mm}$ \\
\hline
\end{tabular}

After the calculation of target points in both vision and thermal camera, one can evaluate the target tracking performance of the eye by comparing standard deviations of the target pixels in $\mathrm{x}$ and $\mathrm{y}$-axis. Nevertheless, this approach does not give the clear indication of the performance. For example, if the variance of the target pixels in the vision camera is along $y$-axis but the variance of the pixels in the thermal camera is along $x$-axis then by comparing the above mentioned parameters does not represent the performance. In such situation, to better evaluate the tracking performance, the principal component analysis (PCA) of the target points can be applied. An example of the PCA of the target points is presented in Figure 10. From the figure, it is clear that the major axis and the minor axis of the PCA represents the direction of maximum and minimum variation of the target points. By using the principal component analysis, the following parameters:

- Maximum eigenvalues of the principal component analysis (PCA) of target pixels
- Minimum eigenvalues of the principal component analysis (PCA) of target pixels

are calculated for evaluation. It should be noted that in this system small variations of the target points in thermal cameras signifies better performance.

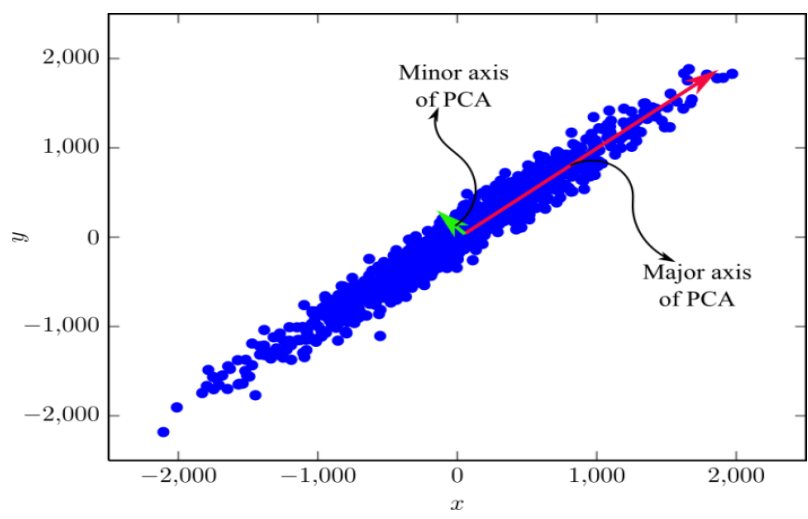

Figure 10. An example of the PCA of target points showing major and minor axis.

From the calculation of the above mentioned parameters, ratios of the parameters are obtained. Table 2 shows the summaries of the performance evaluations. From the table, it can be observed that there are improvements in all parameters. For instance, in terms of ratio of maximum eigenvalues there is the improvement by 74 percent. Similarly, in the case of the ratio of standard deviation in $\mathrm{x}$-axis the improvement is about 42 percent.

Table 2: Summaries of the performance of the thermal tracking system with and without MPC.

\begin{tabular}{cccc}
\hline & $\begin{array}{c}\text { Without } \\
\text { MPC }\end{array}$ & $\begin{array}{c}\text { With } \\
\text { MPC }\end{array}$ & $\begin{array}{c}\% \\
\text { improvement }\end{array}$ \\
\hline $\begin{array}{c}\text { Ratio of } \\
\text { maximum } \\
\text { eigenvalues of } \\
\text { PCA } \\
\text { (thermal/vision) }\end{array}$ & 0.000105 & $27 \times 10^{-5}$ & 74 \\
\hline $\begin{array}{c}\text { Ratio of } \\
\text { minimum } \\
\text { eigenvalues of } \\
\text { PCA } \\
\text { (thermal/vision) }\end{array}$ & 1.2213 & 0.8455 & 30 \\
\hline $\begin{array}{c}\text { Ratio of } \\
\text { standard } \\
\text { deviation in X- } \\
\text { axis } \\
\text { (thermal/vision) }\end{array}$ & & & \\
\hline $\begin{array}{c}\text { Ratio of } \\
\text { standard } \\
\text { deviation } \\
\text { (thermal/vision) }\end{array}$ & 0.0101 & 0.005 & \\
\hline & & & \\
\hline
\end{tabular}




\section{Conclusion}

In this paper, we have presented a thermal target tracking system using RobotEye technology. The system comprises of a robot eye, a thermal camera and a vision camera. Furthermore, the proposed system consists of an estimator for the target orientation and position with an Kalman filter using the application program interface for modeling and a Model Predictive Controller for command generation. In addition, a fiducial marker consisting of circular patches is attached to the target. The objective of the fiducial marker is to estimate the position of marker from the images taken from the vision camera. After the estimation of the target position, its future position is predicted using a predictive filter, and a predictive control is applied to generate command for the eye. Experiments have been conducted and the tracking performance was evaluated for the system, and the improvements of up to 74 percent is observed with the predictive control compared to the non-control case.

\section{Acknowledgement}

This work was conducted at the premises of Ocular Robotics Pty Ltd, and supported by Australian Mathematical Science Institute (AMSI) and Defence Science and Technology Group (DSTG), Department of Defence, Australian Government.

\section{References}

[1] Gade R. and Moeslund T. B., Thermal cameras and applications: a survey, Machine Vision and Applications, 25: 245-262, 2014.

[2] Rogler R.-D., Löbl H., and Schmidt J., A diagnostic system for live electrical joints in power transmission systems, European Transactions on Electrical Power, 7(5), pages 331-336, 1997

[3] Wong, A. K., Dunn S. A., and Sparrow J. G, Residual Stress Measurement by Means of the Thermoelastic Effect. Nature 332, 6165 (1988):

[4] Rajic, Nik, Street N., Brooks C., and Galea S, Full Field Stress Measurement for in Situ Structural Health Monitoring of Airframe Components and Repairs. In 7th European Workshop on Structural Health Monitoring, 2014.

[5] Rajic, N., and Neil S. A Performance Comparison Between Cooled and Uncooled Infrared Detectors for Thermoelastic Stress Analysis. Quantitative InfraRed Thermography Journal 11(2), pages: 207-221 2014.

[6] Rajic N., Brooks C., Wang J., Forrester C., and Swanton G. Thermoelastic Stress Analysis for Structural Performance Assessment of Aerospace Composites, in $21^{\text {st }}$ International Conference on Composite Materials, 2017
[7] Leira F. S., Trnka K., Fossen T. I., and Johansen T. A., A Lightweight Thermal Camera Payload with Georeferencing Capabilities for Small Fixed-wing UAVs, in International Conference on Unmanned Aircraft Systems (ICUAS), pages 485-494, 2015.

[8] Helgesen H. H., Leira F. S., Fossen T. I., and Johansen T. A., Tracking of Ocean Surface Objects from Unmanned Aerial Vehicles with a Pan/Tilt Unit using a Thermal Camera, Journal of Intelligent \& Robotic Systems, 1-19, 2017.

[9] Wood D. and Bishop M., A novel approach to 3D laser scanning, in Proceedings of Australian Conference on Robotics and Automation (ACRA), 2012.

[10] Okumura K., Oku H., and Ishikawa M., Highspeed gaze controller for millisecond-order pan/tilt camera, in IEEE International Conference on Robotics and Automation (ICRA), pages 61866191, 2011.

[11] Singh A. M., Ha Q. P., Wood D. K., and Bishop M., Low-latency Vision-based Fiducial Detection and Localisation for Object Tracking, in 34th International Symposium on Automation and Robotics in Construction pages. 706-711, Taiwan, 2017. 